\title{
A PHOTOGRAMMETRIC PIPELINE FOR THE 3D RECONSTRUCTION OF CASSIS IMAGES ON BOARD EXOMARS TGO
}

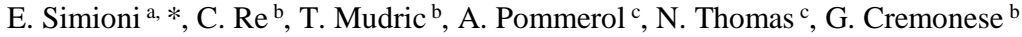 \\ ${ }^{a}$ CNR-Institute for Photonics and Nanotechnologies, Padova LUXOR, Padova, Italy - simioni@dei.unipd.it \\ b INAF-Astronomical Observatory Padova, Padova, Italy (cristina.re, teo.mudric, gabriel.cremonese)@ oapd.inaf.it \\ ${ }^{\mathrm{c}}$ University of Bern, Physikalisches Institut, Space Research and Planetology Division, Bern, \\ Switzerland (antoine.pommerol, nicolas.thomas)@ space.unibe.ch
}

\section{Commission VI, WG VI/4}

KEY WORDS: Image Processing, Satellite Images, Software, Digital Photogrammetry

\begin{abstract}
:
CaSSIS (Colour and Stereo Surface Imaging System) is the stereo imaging system onboard the European Space Agency and ROSCOSMOS ExoMars Trace Gas Orbiter (TGO) that has been launched on 14 March 2016 and entered a Mars elliptical orbit on 19 October 2016. During the first bounded orbits, CaSSIS returned its first multiband images taken on 22 and 26 November 2016. The telescope acquired 11 images, each composed by 30 framelets, of the Martian surface near Hebes Chasma and Noctis Labyrithus regions reaching at closest approach at a distance of $250 \mathrm{~km}$ from the surface. Despite of the eccentricity of this first orbit, CaSSIS has provided one stereo pair with a mean ground resolution of $6 \mathrm{~m}$ from a mean distance of $520 \mathrm{~km}$. The team at the Astronomical Observatory of Padova (OAPD-INAF) is involved into different stereo oriented missions and it is realizing a software for the generation of Digital Terrain Models from the CaSSIS images. The SW will be then adapted also for other projects involving stereo camera systems. To compute accurate 3D models, several sequential methods and tools have been developed. The preliminary pipeline provides: the generation of rectified images from the CaSSIS framelets, a matching core and post-processing methods. The software includes in particular: an automatic tie points detection by the Speeded Up Robust Features (SURF) operator, an initial search for the correspondences through Normalize Cross Correlation (NCC) algorithm and the Adaptive Least Square Matching (LSM) algorithm in a hierarchical approach. This work will show a preliminary DTM generated by the first CaSSIS stereo images.
\end{abstract}

\section{INTRODUCTION}

This paper aims at introducing the stereo processing chain defined by research group located at the Astronomical Observatory of Padova (OAPD-INAF) for the 3D reconstruction of planetary surfaces. The group has been involved in the design of the stereo camera STC [1] for the BepiColombo mission to Mercury whose launch will be in 2018. Since one of the main task is the global stereo coverage of the planet surface; the generation of the global DTM has been the motivation for the development of an ad hoc software for the DTM generation.

The group has been involved even in the realization of CaSSIS, based on the push-frame stereo acquisition, as STC, and having the same detector.

An overview of the CaSSIS telescope is presented in Section 2. Section 3 describes the pipeline (oriented to push-frame cameras).

Two different tests, to validate the stereo chain, are shown in Section 4 while the stereo pipeline applied to the images acquired by CaSSIS during the first orbit around Mars is presented in Section 5 .

\section{CASSIS}

The stereo camera CASSIS will acquire nominally, in the next years, images and stereo pairs of the Martian surface.

\subsection{Instrument overview}

The telescope, a 4-aspheric mirror system, will realize target oriented observations with an on-ground pixel of $4.6 \mathrm{~m}$ from a distance of $400 \mathrm{~km}$.

Thanks to an inclination of $74^{\circ}$ of the orbit, CaSSIS will monitor the daily and seasonal surface changes of the planet.

CaSSIS telescope [2] is mounted on a rotational unit (RU) stage, with an angle respect to the nadir direction of $10^{\circ}$. The RU allows a rotation of $180^{\circ}$.

This configuration allows the instrument to acquire images in the forward and backward direction. CaSSIS is a frame oriented device. This means that the actual 2D images of the planet surface are acquired, then buffered while the spacecraft moves. Only when the on-ground image acquired has shifted along track by an amount corresponding to the FoV of the filter, another image is acquired. A set of thirty framelets are acquired consecutively with an overlapping between 5 and $15 \%$. After these operations, the rotator turns the telescope and a new set of framelets is acquired (see Figure 1). The images composed by framelets have a cross track swat of $9.5 \mathrm{~km}$ and $40 \mathrm{~km}$ along track in the nominal case.

\footnotetext{
* Corresponding author. This is useful to know for communication with the appropriate person in cases with more than one author.
} 

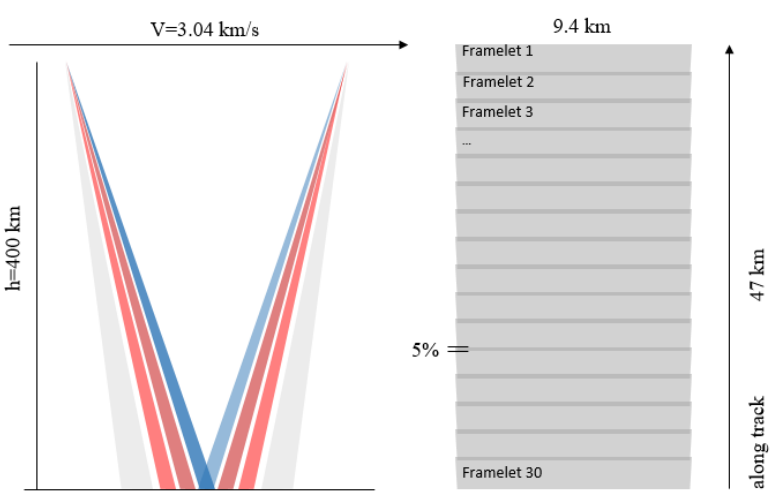

Figure 1 CaSSIS observation strategies. On the left respectively the PAN, RED, NIR, BLU filters FoV for a direct acquisition and after the rotation of the stage. On the right the on-ground footprints in case of broadband filter operations.

\subsection{The first orbit}

Despite of the nominal circular orbit, the first CaSSIS images were not acquired from a constant distance. The TGO, during stereo acquisitions in proximity of the closest approach, was at a distance from surface between 560 and $480 \mathrm{~km}$ with a pixel onground respectively of 6.6 and $5.7 \mathrm{~m}$.

Also from the attitude point of view, the acquisition does not represent the nominal case. The $\mathrm{S} / \mathrm{C}$ attitude, in fact, introduced an incomplete overlapping of the on-ground footprints as shown in Figure 2.

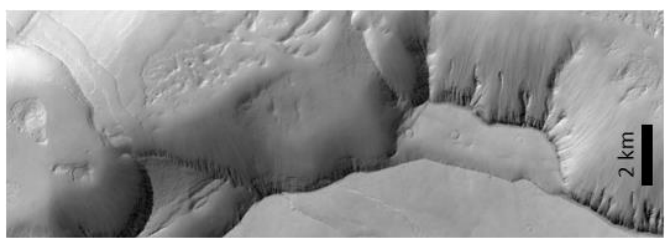

(a)

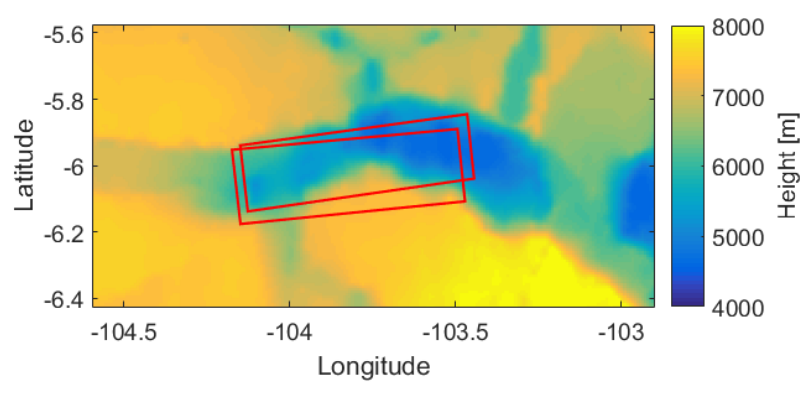

(b)

Figure 2 In (a) a mosaicked image of the first CaSSIS images, in (b) the on-ground footprints of the composed CaSSIS images. The footprints are depicted on the MOLA reference model.

The acquisitions covered the region of Noctis Labyrinthus revealing a Deep Seat Gravitational Slope Deformation (DSGSD)

\section{PIPELINE}

The complete workflow, starting from the CaSSIS framelets up to the final DTM generation is described in Figure 3. The pipeline contains some sequential steps: for the creation of the complete orthoimages from the framelets, for the definition of an initial disparity map, and the disparity refinement at the sub-pixel level, up to the triangulation phase arriving to the DTM production.

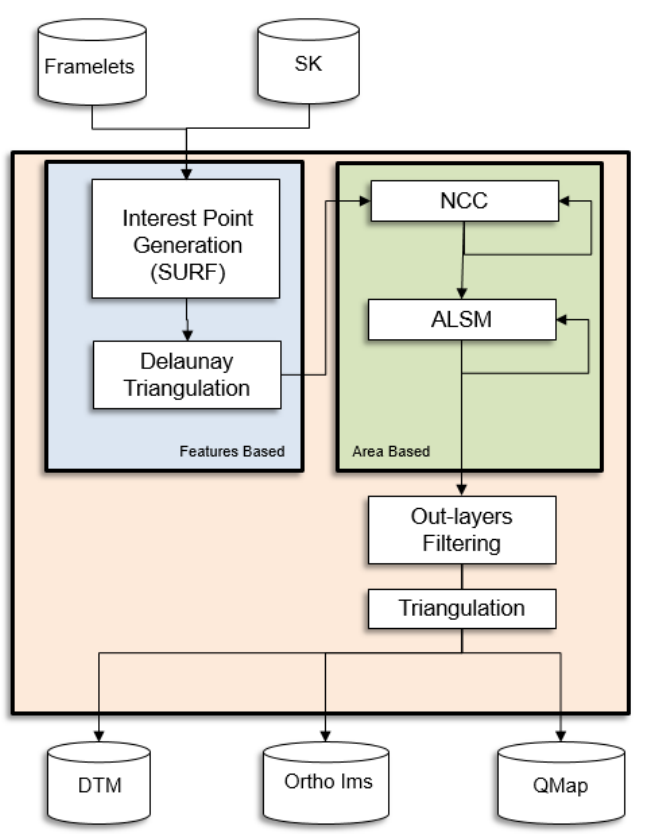

Figure 3 Flow diagram of the processing chain.

The framelets/images accepted in the pipeline can be both in EDR format and/or in the other more common ones (JPG,TIFF,PNG). Even Matlab bin file are welcome.

The generated DTM can be exported in raster format (GeoTIFF) with the corresponding orthophoto; also point clouds and meshes textured (or not) can be provided.

The architecture of the data storage is matrix based: the disparity and quality maps are defined as float jarred array.

This approach has been chosen to guarantee a performant exploitation for all the intermediate products in the pipeline and to make easier the user interactions during the procedures (such as filtering)..The strategy makes the pipeline easily extendible to different complementary algorithms and techniques (such as semi global or shape from shading).

\subsection{Mosaicking SURF based}

The Spice Kernels provided, for this first orbit, the interior camera parameters and an approximated definition for the external orientation parameters, describing the instantaneous position and attitude of the telescope and of each framelet. After the distortion removal (considering its preliminary definition), a fast strategy that exploits the stereo geometry of the acquisitions has been implemented to obtain the best stereo rectified image pair to be processed. 
Images have been rectified projecting them on a plane at a height identified by the triangulation process of a set of tie points extracted thanks to the SURF [[4] interest operator.

The process is as follows:

(i) calculation of the mean surface height $\mathrm{H}$ with respect to the orbit by using the Spice Kernels.

(ii) projection of the framelets on a plane at the distance $\mathrm{H}$.

(iii) definition of a set of tie-points (correlated) on both the images by using the SURF operator

(iv) estimation of the actual mean height $\mathrm{H}$ from the triangulation of the tie points

(v) iteration of the point coordinates extraction until $\mathrm{H}$ achieve a stable value.

This bundle adjustment strategy allows to obtain the best mosaicked images avoiding misalignments along track between framelets which can achieve 1 pixel in case the height of the projection surface differs of $2.2 \mathrm{~km}$ with respect to the real position of the target.

The images are resampled through a bilinear interpolation. In the overlapping regions (Figure 4) where the parallax error is minimized, the intensity values correspond to a weighted mean between the overlapping framelets. The weight factor changes according to a r-cosine function from 1 to 0 in the along-track direction (Figure 4.b).

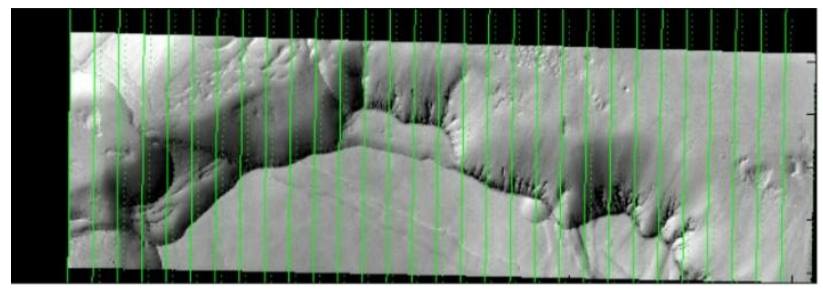

(a)

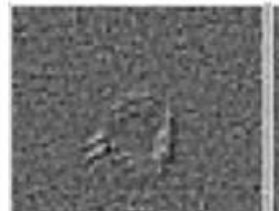

$H_{1}$

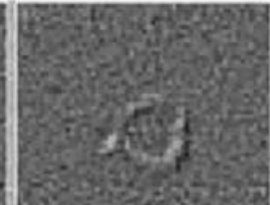

$\mathrm{H}_{2}$

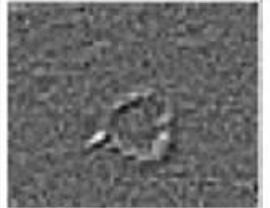

$\mathrm{H}_{3}$ (b)

Figure 4 In (a) mosaicking from CaSSIS first stereo framelets. In (b) the Laplacian filter applied to an overlapping zone mosaicked for consecutive repetitions of the procedure described, the best alignment is reached at third iteration.

\subsection{Matching phase}

The pipeline proposed for the CASSIS images include three fundamental stages:

(i) a feature based extraction of the seed points for the definition of a starting location of the homologous points.

(ii) a parabola fitting normalized cross correlation for the initial search for the correspondences

(iii) a Least Squares Matching [5],[6] subpixel refinement.
The workflow starts considering a number of seed (tie) points. Through the Delaunay triangulation [[7] and the consequent interpolation, the starting location of the corresponding points on the slave image are determined and an approximate parallax field is defined.

Then, an initial search for the correspondences through NCC algorithm (accelerated using summing tables [8]) is performed starting from the previous parallax values. Then the disparity map is refined by the LSM.

A 2D parabola fitting of the matching cost is applied to reduce the pixel locking effect.

The resulting disparity map is then improved using a ALSM to achieve the sub-pixel accuracy.

A pyramidal structure is defined allowing to apply the NCC and the ALSM algorithms at the different resolution levels.

The code is implemented in order to define through the MAtrix User Interface (MAUI) different parameters such as: window template dimension, search area dimension, number of pyramidal levels, similarity functions, outlier thresholds, ALSM iterations number.

The same MAUI allows to have a statistical and visual representation of all the intermediate data as matrix structures: the images, the corresponding statistical indexes (local standard deviation, local contrast, or gradients), the disparity maps as well as the quality maps (described by the similarity function).

\subsection{Outliers rejection}

Since the correlation process can encounter difficulties, the final results can be affected by a number of mismatches and blunders. The outliers can be detected in different ways:

- the thresholds on the NCC

- the statistical bound on the disparity maps and on its gradient

- threshold on the distance from the local mean (to avoid spike effect)

These user-defined methods allow at each stage of the pipeline to define the fail matching regions and preserve the continuity on the surface.

\section{TESTS}

The Pipeline was tested on the first stereo pair acquired by CaSSIS. Furthermore, two more tests were performed for validation:

(i) HERSHEL_CRATER: based on a pair of synthetic images generated by using the HiRISE Texturized DTM.

(ii) STC_SVS: based on a couple of images acquired during SIMBIO-SYS STC stereo calibration on-ground phase.

Main details and results of the tests are described in the following subsections. 


\subsection{Hershel-Crater}

To generate the synthetic images, a DTM produced by the University of Arizona (UA) with Socet Set SW (@BAE Systems) [9] starting from the stereo pairs of HiRISE

[10] has been imported in Blender [11] This software is a free and open source $3 \mathrm{D}$ creation suite which supports raytracing rendering. Blender allows to define and to include cameras in the scene, to overlay objects with texture and to control illumination and generate synthetic images of the scene [12].

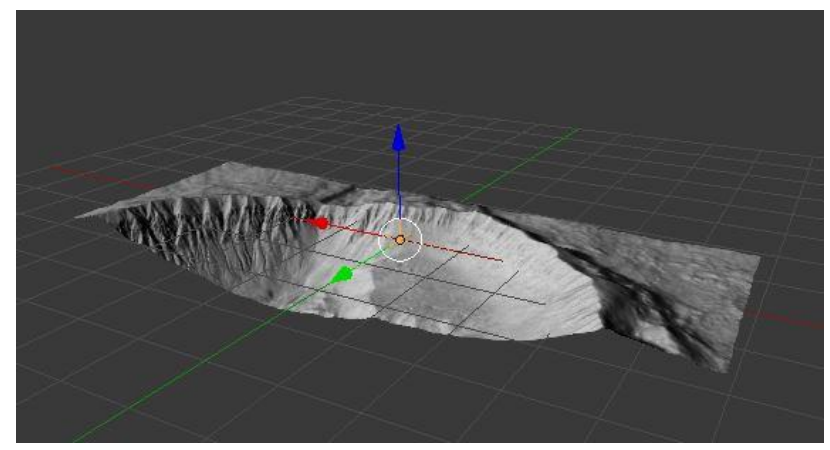

Figure 5 HiRiSe DTM of the Herschel Crater

Two virtual cameras that simulate CaSSIS (in terms of intrinsic parameters) have produced images of the artificial scene with the near nominal acquisition block geometry of the telescope. Images dimensions are 500x2048 pixel.

For sake of simplicity, no optical distortion was applied to the images. On the other side, the images are rendered at 10 times the required resolution, introducing an artificial PSF of 1.25 pixels convolved with the images which are then sampled to final resolution.

A white noise of $10 \mathrm{DN}$ is added to the images to simulate Read Out Noise (RON).

Images have a different pixel scale: the two virtual cameras representing CaSSIS are disposed at a mean distance of $422 \mathrm{~km}$ from the surface with a difference of $11 \%$ between the two acquisitions and a maximal scale of $5.2 \mathrm{~m} / \mathrm{px}$. An additional rotation was introduced around boresight axis (Figure 6).

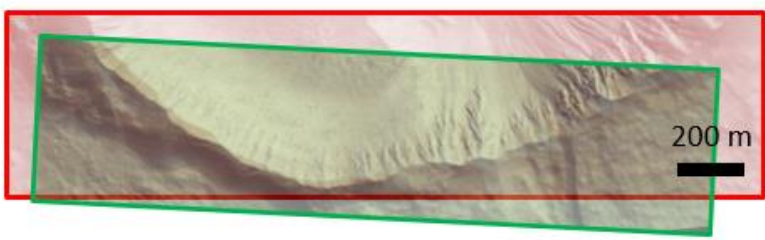

Figure 6 Overlapped images of the Hershel Crater Test.

Using such synthetic images and the orientation data, a point cloud has been generated by a dense image matching and the final triangulation; the reconstruction error in the object space can be evaluated point-wise as the distance of each point from the reference DTM.

The reference DTM (UA-HiRISE) has been fixed and the DTM reconstruction has been aligned through a 3D modelling software. A threshold of $30 \mathrm{~m}$, has been considered in order to reject the outliers from the comparison.
Comparison results are shown in Table 1.

\begin{tabular}{|l|c|}
\hline Data & Hershel Crater \\
\hline Reference & HiRiSe DTM. \\
Pixel on-ground & $5.2 \mathrm{~m}$ \\
Threshold & $30 \mathrm{~m}$ \\
\# Completeness & $91 \%$ \\
Mean & $0.03 \mathrm{~m}$ \\
RMS & $1.1 \mathrm{~m}$ \\
\hline
\end{tabular}

Table 1 Reconstruction performance of the SW in HERSCHEL_CRATER test.

The acquisition block geometry introduces a Vertical Accuracy of 14.9 metres (considering a parallax error of $1 \mathrm{px}$ ) and the software reaches a matching error of $0.1 \mathrm{px}$, as expected for synthetic images [13].

\subsection{STC-SVS, images for stereo validation}

The STC system is a Stereo Camera on board of ESA-JAXA BepiColombo mission [1], that will be launched in 2018 toward Mercury planet.

STC and CaSSIS are both facing to surface reconstruction and color acquisition but they are characterized by a different design and concept.

STC is, in fact, characterized by two optical channels inclined both of $20 \mathrm{deg}$ with respect to the nadir direction [14] looking in forward and backward directions.

Both the channels share a common telescope unit and the detector, a SiPin CMOS (same adopted by CaSSIS) which guarantees a fill factor of $100 \%$ thanks to a Back-Side Illumination (BSI) configuration; a focal length of $95.2 \mathrm{~mm}$ brought the telescope performance to achieve an on-ground pixel of $58 \mathrm{~m}$ (at $480 \mathrm{~km}$ from the surface in the first orbit).

To test the capabilities of the photogrammetric chain we take advantage of the on-ground STC images: during the calibration campaign of the telescope, in fact, a Stereo Validation Setup (SVS) [16] has been conceived and realized in laboratory in order to validate the new push frame concept of STC [[16].

Basically, a set of images have been acquired in the limited ambient of the clean room with the flight model of STC by means of a collimator lens and two motorized rotators which has reproduced the stereo block geometry of STC operations.

The images acquired with the SVS have been used as test for the pipeline of the SW.

In order to evaluate the accuracy of the $3 \mathrm{D}$ products, an high resolution laser scanning DTM has been used as reference. 


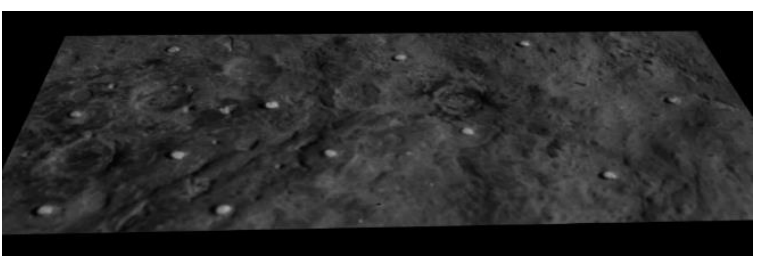

(a)

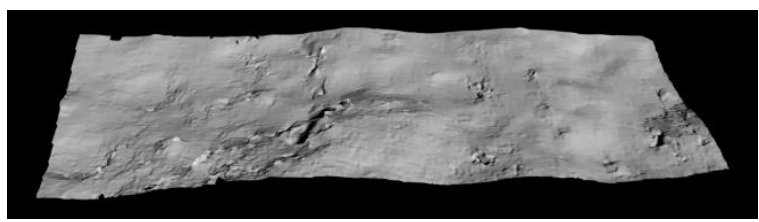

(b)

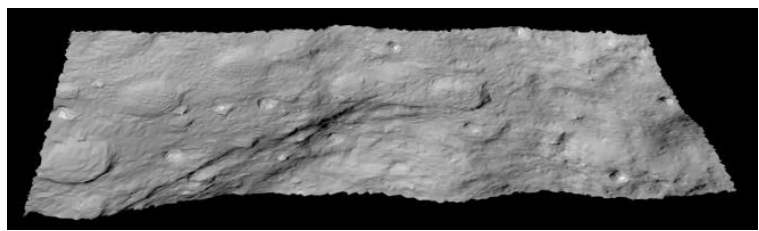

(c)

Figure 7 In the top panel (a) we show the Image obtained by one STC channel of a stone target. In the middle panel (b) the DTM, generated by the pipeline is shown and in the bottom panel the DTM generated by the Laser scanner (c).

The performance of the SW to provide an accurate 3D reconstruction of surfaces has been evaluated using the STC stereo pairs bench also as test. A reference DTM of the rock samples has been provided by a CAM2® FaroArm Platinum scanner system with a $Z$ axis accuracy of $0.02 \mathrm{~mm}$. The highresolution laser scanner reconstruction of the sample target has a better vertical accuracy than the one provided by the stereo image reconstruction and has therefore been used as "true" data. The comparison between the stereo DTM and the reference one gave the confidence about the capability of the system.

As quality figures for the results, the percentile of accepted matches and the Root Mean Square Error (RMSE) computed as RMS of the absolute distance from the reference surface of each point have been considered.

\begin{tabular}{|l|c|}
\hline Data & STC DTM \\
\hline Reference & Laser Acq. \\
Threshold & $0.5 \mathrm{~mm}$ \\
\#Completeness & $99.6 \%$ \\
Mean & $1.9 \mu \mathrm{m}$ \\
RMS Error & $73 \mu \mathrm{m}$ \\
\hline
\end{tabular}

Table 2 Reconstruction performance of the SW in STC Images.

Table 2 reports the results of the comparison. As far as the accuracy of reconstruction is concerned, the obtained RMS values are all well below the instrument scientific requirements at the breadboard scale (with a pixel scale of $105 \mu \mathrm{m}$ ).

Considering the scale factor between the SVS and the nominal operative configuration of STC the Vertical Precision obtained corresponds to $36 \mathrm{~m}$ (taking in to account a on ground pixel of 58 metres at first orbit around Mercury).

\section{CASSIS FIRST ORBIT}

In the case of CaSSIS images, the not nominal block geometry configuration made the overlapping between the images not sufficient to provide a features based alignment of each framelets with the followings. Anyway, a Bundle Adjustment strategy on the stereo couples makes possible a minimal correction of the camera pointing (in the tolerance limits of the calibration).

At the same time, the preliminary definition of the distortion map of the instrument has been applied before the application of the procedure described in the 3.1 paragraph

The implemented strategy allowed to define a global and easy projection able to provide a stereo pair to be processed in the photogrammetric pipeline.

Considering the surrender to the map-projection approach, the preliminary definition of the camera model (not updated in the SPICE kernels yet), the method described in Section 3.1 for the mosaicking of the framelets provided images $(25 \%$ of the instrument resolution) with no misalignment between the framelets.

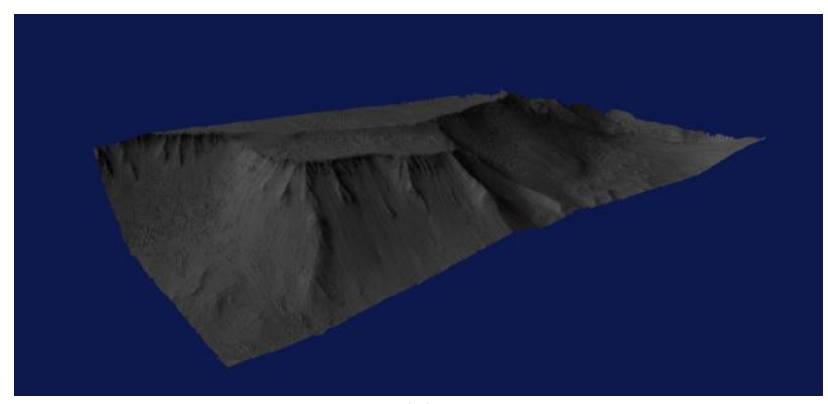

(a)

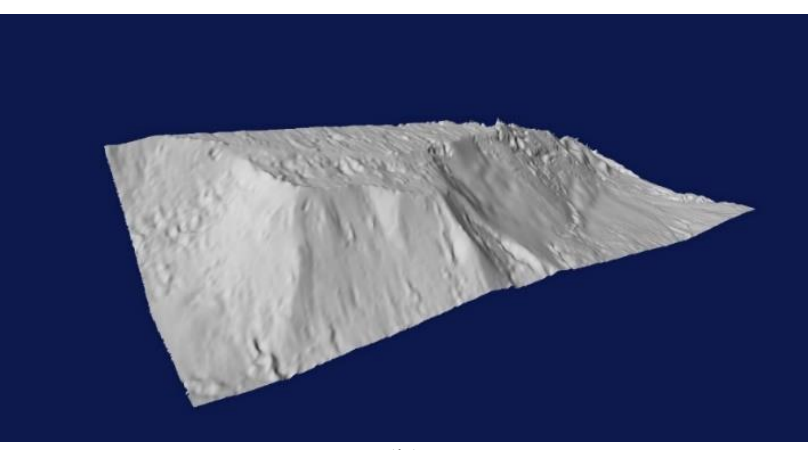

(b)

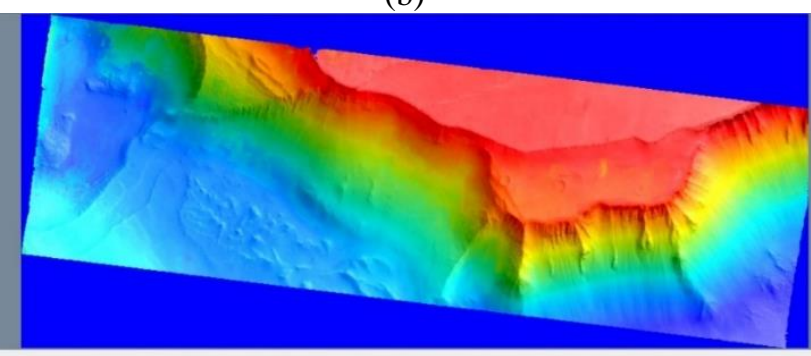

(c)

Figure 8 In the top panel (a) and (b) we showed the DTM reconstruction respectively texturized and as simple mesh. In (c) the color-mapped reconstruction snapshot of the MUI. Colormap is limited by $6-8.1 \mathrm{~km}$. 
Different instruments of the past missions to Mars covered the same region on the surface: the laser-altimeter MOLA[17] and two push-broom telescopes (HRSC [18] and CTX [19]) have been taken in account.

Table 3 shows the 3D remote sensing products considered in this analysis.

\begin{tabular}{|l|c|c|c|}
\hline Instrument & Mission & $\begin{array}{l}\text { Stereo } \\
\text { Pipeline }\end{array}$ & $\begin{array}{c}\text { Resolution } \\
{[\mathbf{m}]}\end{array}$ \\
\hline CASSIS & ExoMars 2016 & OAPD & 28 \\
CTX & MRO & ASP & 5.56 \\
HRSC & Mars Express & DLR & 125 \\
MOLA & MGS & - & 1850 \\
\hline
\end{tabular}

Table 3. DTM instrument sources taken in account for the analysis of the same CaSSIS region.

The DTMs provided by the three instruments are shown in Figure 9 using MOLA data as base map. The CaSSIS model reaches the best details definitions.

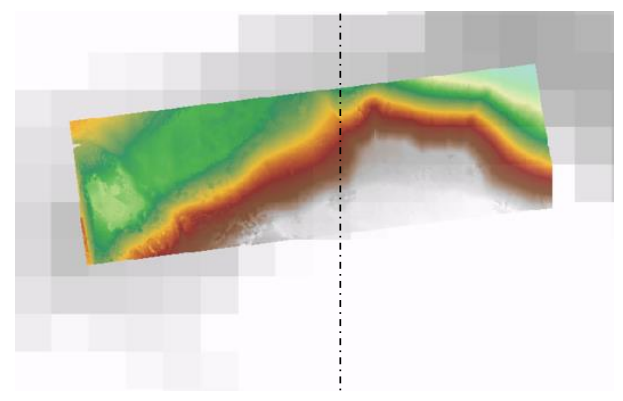

(a)

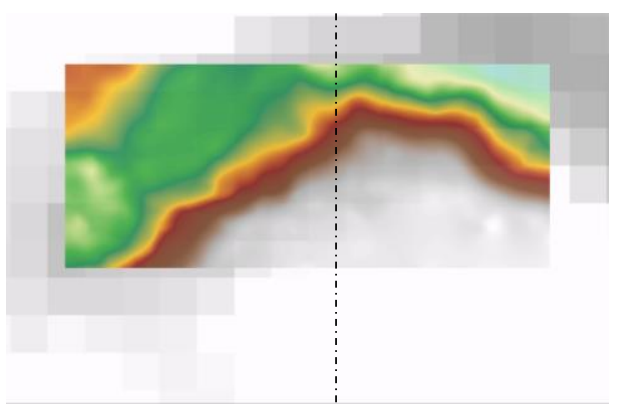

(b)

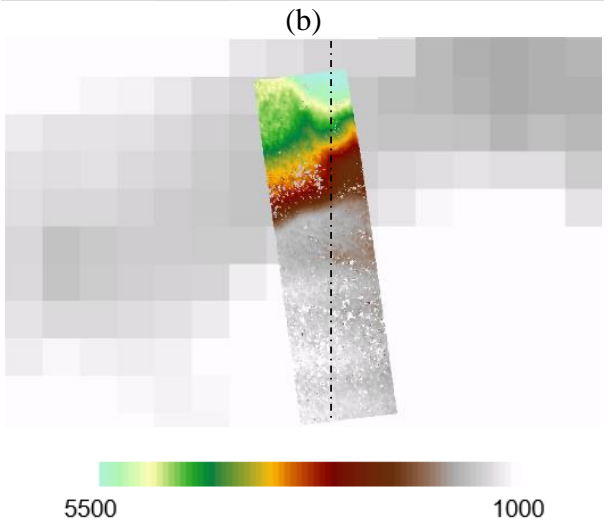

(c)
Figure 9 The three DTM covering the region taken in consideration. In (a) the 3D reconstruction performed by OAPD Pipeline on CaSSIS images; in (b) the HRSC dataset; in (c) the CTX -DTM. Dotted line defines the meridian analysed in comparing profiles.

The CTX images have a spatial resolution comparable with the CaSSIS ones. The CTX DTM (Figure 9) has been generated by a photogrammetric pipeline that considers a pre-processing phase with ISIS3 and the matching process has been performed with Ames Stereo Pipeline (ASP) [[20].

A comparing profile section of the meridian at $-103.79^{\circ}$ (vertical dotted line in the figure) for the three DTMs aligned is shown in the next plot (Figure 10).

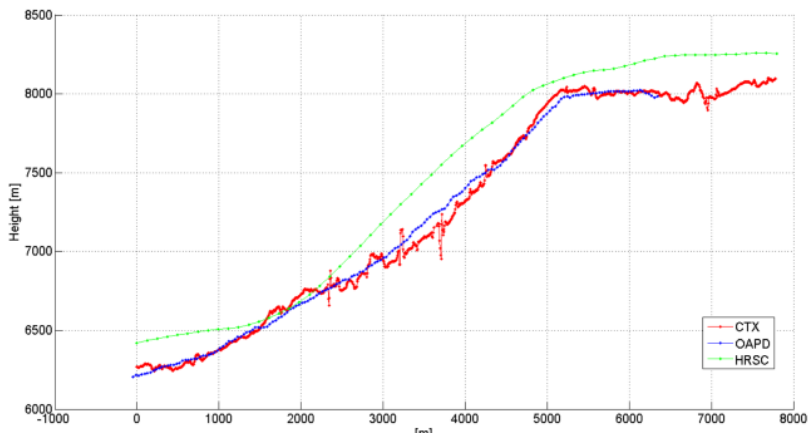

Figure 10 A vertical profile line was used as slice definer of the reconstructions.

Despite the comparable spatial resolution of the CTX images, the observing conditions were not so good: several regions on the frames are characterized by image saturation, the block geometry presents a small baseline of $10.1 \mathrm{~km}$ on a height of $260 \mathrm{~km}$ and a quite strong difference in the slope variation and so also in the perspective within the stereo pair. The comparison between the DTMs is not easy.

\section{CONCLUSIONS}

A photogrammetric stereo processing workflow has been proposed for the 3D reconstruction from CaSSIS images. The pipeline includes a procedure for the pre-processing image management and different approaches for the stereo image matching. Two different tests based on synthetic and real images are presented to validate the pipeline. At the same time, the first stereo images acquired by the CaSSIS telescope have been analysed to achieve the better accuracy even considering a preliminary distortion map and the exploitation of the first version of the Spice Kernels data.

The tests have demonstrated the capabilities of the SW to provide a three-dimensional product from the CaSSIS stereo images by implementing several methods and tools that will be useful for the generation of the DTMs during the nominal acquisition of the camera during the orbiting around Mars.

New distortion model of the telescope [[21] and Frame Kernels definition are still in improvement. Better results will be provide after this updates.

\section{References:}


[1] Cremonese, Gabriele, et al. "The stereo camera on the BepiColombo ESA/JAXA mission: a novel approach." Advances in Geosciences 15 (2009): 305.

[2] Thomas, Nicolas, and Gabriele Cremonese. "The Colour and Stereo Surface Imaging System for ESA's Trace Gas Orbiter." EGU General Assembly Conference Abstracts. Vol. 18. 2016.

[3] Smith, David E., et al. Mars Orbiter Laser Altimeter: Experiment summary after the first year of global mapping of Mars. Journal of Geophysical Research: Planets 106.E10 (2001): 23689-23722.

[4] Bay, H. et al., 2008. Speeded-up robust features (SURF). Computer vision and image understanding 110.3: pp. 346-359.

[5] Gruen, Armin. Adaptive least squares correlation: a powerful image matching technique. South African Journal of Photogrammetry, Remote Sensing and Cartography 14.3 (1985): 175-187.

[6] Baker, Simon, and Iain Matthews. Lucas-kanade 20 years on: A unifying framework. International journal of computer vision 56.3 (2004): 221-255.

[7] C. L. Lawson, "Software for C surface interpolation," in Mathematical Software III, J. Rice, Ed. (Academic Press, New York, 1977).

[8] Lewis, John P. "Fast normalized cross-correlation." Vision interface. Vol. 10. No. 1. 1995.

[9] Miller, S. B., and A. S. Walker, Die Entwicklung der digitalen photogrammetrischen Systeme von Leica und Helava, Z. Photogramm. Fernerkundung,1(95), 4 -16, 1995.

[10] McEwen, Alfred S., et al. "Mars reconnaissance orbiter's high resolution imaging science experiment (HiRISE)." Journal of Geophysical Research: Planets 112.E5 (2007).

[11] Pieters, Carle M., et al. "Composition of surface materials on the moons of Mars." Planetary and space science 102 (2014): 144-151.

[12] Re, C., Roncella, R., Forlani, G., Cremonese, G., Naletto, G., 2012. Evaluation of area based image matching applied to DTM generation with Hirise images. ISPRS Ann.Photogramm. Remote Sens. Spat. Inf. Sci. Vol I-4, 209-214.

[13] Gruen, Armin. "Development and status of image matching in photogrammetry." The Photogrammetric Record 27.137 (2012): 36-57.

[14] Da Deppo, V., et al. "Preliminary results of the optical calibration for the Stereo Camera STC onboard the BepiColombo mission." International Conference on Space Optics. Vol. 7. 2014.

[15] Simioni E., et al. "Stereo Camera for satellite application: A new testing method." Metrology for Aerospace (MetroAeroSpace), 2014 IEEE., 2014.

[16] Re, C., et al. "Effects of image compression and illumination on digital terrain models for the stereo camera of the
BepiColombo mission." Planetary and Space Science 136 (2017): 1-14.

[17] Smith, D.E. et al."Mars Orbiter LaserAltimeterExperiment summary after the first year of global mapping of Mars" Journal of Geophysical Research, v. 106, no. E10, p. 23,689-23,722. 2001

[18] Jaumann, R., et al. "The High Resolution Stereo Camera (HRSC): 10 Years of Imaging Mars. Eighth International Conference on Mars. Vol. 1791. 2014.

[19] Malin, Michael C., et al. "Context camera investigation on board the Mars Reconnaissance Orbiter." Journal of Geophysical Research: Planets 112.E5 (2007).

[20] Moratto, Z. M., et al. "Ames Stereo Pipeline, NASA's open source automated stereogrammetry software." Lunar and Planetary Science Conference. Vol. 41. 2010.

[21] Tulyakov S. et al, "Geometric calibration of Colour and Stereo Surface Imaging System of ESA's Trace Gas Orbiter" Advances in Space Research submitted arXiv: 1707.00606. 